\title{
3D modeling and 3D printing in functional urology: the future perspective
}

\author{
Emre Huri $^{1}$ - Sherif Mourad ${ }^{2}$ - Alka Bhide ${ }^{3}$ - Giuseppe Alessandro Digesu ${ }^{3}$
}

Received: 13 March 2020 / Accepted: 13 March 2020 / Published online: 2 April 2020

(C) The International Urogynecological Association 2020

We know that anatomical abnormalities in the pelvic region may cause various lower urinary tract symptoms and pelvic floor disorders such as stress incontinence, urge incontinence, urgency, and pelvic organ prolapse. These structures create both static and dynamic anatomy that involves pelvic organs, muscles, fascial layers, nerves, vessels, ligaments, and connective tissue. Damage to these structures results in pelvic floor disorders [1-3].

It is known that all structures in the pelvis are covered by a fascial layer arising from the pelvic wall. This fascial layer envelops nerves and vessels as well. The correction or substitution of these structures should be the main target in functional and reconstructive pelvic floor surgery. In clinical practice, we use patient-specific CT or MRI reconstructed 3dimensional (3D) modeling and 3D printing technology for a better understanding of 3D surgical anatomy, increasing visibility for hard-to-see structures. This allows completion of virtual surgical procedures, surgical planning, patient and student education, and the creation of hands-on training material for residents [4-7]. Obviously, if you see a more detailed anatomy, you will perform surgery better. 3D systems enhance your perception of the 3D visualization of organs and surgical fields. That is why this technology may be very helpful in pelvic-perineal surgeries, which have complex anatomy in both male and female patients. In our daily clinical practice, CT or MRI scans give us very important clues before the surgery in Digital Imaging and Communications in Medicine (DICOM) format. The basis of 3D printing technology is the $3 \mathrm{D}$ modeling of organs and anatomical structures

Emre Huri

emrehuri@hacettepe.edu.tr

1 Faculty of Medicine, Urology Department, Hacettepe University, Ankara, Turkey

2 Ain Shams University, Cairo, Egypt

3 Imperial College London, London, UK using DICOM. Therefore, we should take into account how we can take reconstruction data from 2-dimensional (2D) images to create $3 \mathrm{D}$ geometric models, which requires proper software and hardware. Medical images from hospitals consist of a $2 \mathrm{D}$ dataset and provide human body information as a slice. The human body has morphological structures in a 3D space. To recognize real human organs, the body should be reconstructed using $2 \mathrm{D}$ slices to obtain its precise position and shape. With medical 3D data, we can obtain more information about the human body, as well as more objective data from the simulation, which may contribute to more successful treatment and surgery plans. If we can simulate this 3D morphology, we may be able to obtain more information about the body and contribute to the clinical environment for both treatment and surgical outcomes. From modeling to the 3D printing process, there are some steps that need to be followed: modeling, visualization, data management, simulation, and printing. If you search 3D printing applications in functional urology and urogynecology in the literature, there are very few articles or reports on this topic compared with other surgical disciplines such as plastic surgery, otolaryngology, and orthopedic surgery. A recent European Union project, named "MedTRain3DModsim," used 3D applications for education in urological surgery. The MedTRain3DModsim Project was aimed at producing 3D medical printed models, simulations, and innovative applications for every level of medical training using novel worldwide technologies. A 3D printed pelvic surgery model and a 3D printed sacral neuromodulation model created using real patient radiological data seem to be as effective as cadaveric models for surgical skills training $[8,9]$.

The benefits of 3D printing models produced using presurgical CT scans, 3D modeling, and 3D printing technology are: improvement in surgical anatomy and technique, surgical planning for complex cases, bioprinted tissue reconstruction for prosthesis surgery, and resident and patient education.

In conclusion, 3D printing is an evolving technology that enables the creation of unique organic and inorganic structures with high precision. In urogynecology as well as functional 
urology, the technology has demonstrated potential uses in both patient and clinician education, as well as in clinical practice $[8,9]$. However, as a clinician, we should be aware of the potential errors of 3D printing technology, and the basics of patient-centered clinical decision-making should always be paramount. The reason for use, collaboration with engineers and mentoring them, detailed anatomical knowledge, post-processing management of 3D printed models, standardization and validation, cost-effectivity, and re-usable format are all important landmarks for the professional clinical applications of $3 \mathrm{D}$ printing.

\section{Compliance with ethical standards}

Financial disclaimer None.

Conflicts of interest None.

\section{References}

1. Urbankova I, Grohregin K, Hanacek J, Krcmar M, Feyereisl J, Deprest J, et al. The effect of the first vaginal birth on pelvic floor anatomy and dysfunction. Int Urogynecol J. 2019;30(10):1689-96. https://doi.org/10.1007/s00192-019-04044-2.

2. Dietz HP, Scoti F, Subramaniam N, Friedman T, Shek KL. Impact of subsequent pregnancies on pelvic floor functional anatomy. Int Urogynecol J. 2018;29(10):1517-22. https://doi.org/10.1007/ s00192-018-3567-9.
3. Jean Dit Gautier E, Mayeur O, Lepage J, Brieu M, Cosson M, Rubod C. Pregnancy impact on uterosacral ligament and pelvic muscles using a 3D numerical and finite element model: preliminary results. Int Urogynecol J. 2018;29(3):425-30. https://doi.org/10.1007/ s00192-017-3520-3.

4. Mahoney C, Hindle A, Rajashanker B, Kearney R. MR scan evaluation of pelvic organ prolapse mesh complications and agreement with intra-operative findings. Int Urogynecol J. 2019. https://doi. org/10.1007/s00192-019-04182-7.

5. Wyman AM, Salemi JL, Mikhail E, Bassaly R, Greene KA, Hart S, et al. Cost-effectiveness of a preoperative pelvic MRI in pelvic organ prolapse surgery. Int Urogynecol J. 2019. https://doi.org/10.1007/ s00192-019-04089-3.

6. Huebner M, DeLancey JOL. Levels of pelvic floor support: what do they look like on magnetic resonance imaging? Int Urogynecol J. 2019;30(9):1593-5. https://doi.org/10.1007/s00192-019-03986-x.

7. Grob ATM, Olde Heuvel J, Futterer JJ, Massop D, Veenstra van Nieuwenhoven AL, FFJ S, et al. Underestimation of pelvic organ prolapse in the supine straining position, based on magnetic resonance imaging findings. Int Urogynecol J. 2019;30(11):1939-44. https://doi.org/10.1007/s00192-018-03862-0.

8. Berger AA, Abramowitch S, Moalli PA. 3D vascular anatomy of the presacral space: impact of age and adiposity. Int Urogynecol J. 2019;30(3):401-7. https://doi.org/10.1007/s00192-017-3542-x.

9. Albanesi G, Giannini A, Carbone M, Russo E, Mannella P, Ferrari V, et al. Computed-tomography image segmentation and 3Dreconstruction of the female pelvis for the preoperative planning of sacrocolpopexy: preliminary data. Int Urogynecol J. 2019;30(5): 725-31. https://doi.org/10.1007/s00192-018-3706-3.

Publisher's note Springer Nature remains neutral with regard to jurisdictional claims in published maps and institutional affiliations. 